\title{
Nigella sativa seed extract attenuates the fatigue induced by exhaustive swimming in rats
}

\author{
MAHBUBUR RAHMAN $^{1 *}$, DONG KWON YANG ${ }^{1 *}$, GI-BEUM KIM $^{2}$, SEI-JIN LEE ${ }^{3}$ and SHANG-JIN KIM ${ }^{1}$ \\ ${ }^{1}$ Department of Veterinary Pharmacology and Toxicology, College of Veterinary Medicine, Chonbuk National University, \\ Iksan-si, Jeollabuk-do 54596; ${ }^{2}$ Institute of Jinan Red Ginseng, Jinan-eup, Jinan-gun 55442; \\ ${ }^{3}$ Korea Basic Science Institute Jeonju Center, Deokjin-gu, Jeonju-si, Jeollabuk-do 54896, Republic of Korea
}

Received December 30, 2016; Accepted February 13, 2017

DOI: $10.3892 /$ br.2017.866

\begin{abstract}
In previous studies, Nigella sativa (NS) has been studied due to its various physiological and pharmacological activities. However, evidence on the effects of NS on physical fatigue following exhaustive swimming remains limited. In the present study, the authors evaluated the potential beneficial effects of NS against the fatigue activity following exhaustive swimming. Rats were orally administered with NS extract ( $2 \mathrm{~g} / \mathrm{kg} /$ day) for 21 days, and the anti-fatigue effect was assessed by exhaustive swimming exercise. The presented results indicated that pre-treatment of NS extract significantly increased the time to exhaustion. In hemodynamic parameters, NS extract increased blood $\mathrm{pO}_{2}$ and $\mathrm{O}_{2 \text { sat }}$, but decreased $p \mathrm{CO}_{2}$. For underlying mechanisms, NS extract protected depletion of energy, indicated by increased levels of blood $\mathrm{pH}$, glucose and tissue glycogen contents, and decreased levels of blood lactate, tissue lactic dehydrogenase and creatine kinase, when the NS extract was pre-treated. In addition, the NS extract inhibited oxidative stress following exhaustive swimming, as reflected by the results of increased levels of superoxide dismutase and redox ratio, and decreased the level of malondialdehyde when the NS extract was pre-treated. Collectively, the present study demonstrated that NS extract has an anti-fatigue activity against exhaustive swimming by energy restoration and oxidative-stress defense.
\end{abstract}

\footnotetext{
Correspondence to: Dr Shang-Jin Kim, Department of Veterinary Pharmacology and Toxicology, College of Veterinary Medicine, Chonbuk National University, Iksan Campus, 79 Gobong-ro, Iksan-si, Jeollabuk-do 54596, Republic of Korea

E-mail: abbasj@jbnu.ac.kr

*Contributed equally
}

Key words: Nigella sativa, fatigue, exhaustive swimming, energy depletion, oxidative stress

\section{Introduction}

Nigella sativa (NS) seed, commonly known as a black cumin, belongs to the Ranunculaceae family (1). It is an herbaceous plant, which is composed of several constituents, including moisture, oil, proteins, carbohydrates, vitamins and minerals (2,3). Among these, fixed oils and unsaturated fatty acids comprise 30 and $85 \%$ of the ingredients in NS seed $(2,4)$. In addition, NS seed contains various chemical compounds with pharmacological properties, including thymoquinone, nigellone, melanthin, damascenone, $\mathrm{p}$-cymene and pinene (5-7). NS seed has been traditionally used as a folk medicine in North Africa, Southeast Asia and Mediterranean countries for many centuries. Indeed, it is used for the treatment of asthma, bronchitis, cough, fever and headaches (8). Previous studies have demonstrated that NS seed has numerous therapeutic activities, such as anti-inflammatory (9), antioxidant $(5,10)$, immune-modulatory $(11)$, cardioprotective $(12,13)$ and hepatoprotective effects (14). However, the effects of NS seed on the fatigue following exercise have not yet been fully elucidated.

Fatigue is defined as a feeling of extreme physical or mental tiredness, resulting from severe stress and hard physical or mental work $(15,16)$. It may be associated with many activities, such as exercise, aging, tumor growth, multiple sclerosis and Parkinson's disease (17-19). In particular, physical fatigue induced by strenuous exercise is thought to lead to a deterioration in performance, causing a decrease in muscular power and endurance, as well as in mental functions $(20,21)$. The underlying mechanism suggests that strenuous exercise accumulates metabolic products (e.g., reactive oxygen species (ROS), lipid peroxides and lactic acid), and leads to oxidative stress, which can potentially contribute to fatigue $(22,23)$.

Accordingly, many studies have focused on the development of drugs or therapies with respect to fatigue $(24,25)$. In addition, considering the limitations of available therapies for fatigue in modern medicine, potential alternatives from traditional medicine are worth investing because of their safety, availability, and ease of administration (26). In the current study, the authors sought to investigate the anti-fatigue activities of NS seed extract in rats subjected to the exhaustive swimming test as a fatigue model. 


\section{Materials and methods}

Preparation of NS extract. NS seeds were obtained from a local market in Mymensingh, Bangladesh. The extraction of NS seeds was performed as previously described (27). Briefly, the black seeds were washed with running tap water, air-dried and ground to powder form $(0.2-0.3 \mathrm{~mm}$ particle size, to ensure homogeneity). A total of $800 \mathrm{~g}$ powdered seeds were extracted in a Soxhlet apparatus with petroleum ether. The extract obtained was evaporated to a viscous liquid at $40^{\circ} \mathrm{C}$ under reduced pressure (yield of $300 \mathrm{ml}, 310.7 \mathrm{~g}$ and 39.2\%). Gas chromatographic analysis of the final extract failed to identify significant amounts of $n$-hexane and $n$-heptane $(0.1 \%)$, which are the major indicators for this distillate.

Animal models and NS seed extract administration. All experimental protocols employed herein were approved by the Committee on the Care of Laboratory Animal Resources of ChonbukNational University and were conducted in accordance with the Guide for the Care and Use of Laboratory Animals published by the US National Institutes of Health (Bethesda, MA, USA; NIH Publication no. 85-23, revised 1996). A total of forty male Sprague-Dawley rats (220-250 g, Samtako Bio Korea Co., Ltd., Daejeon, Korea) were used for all experiments. Animals were housed in cages maintained at $23 \pm 2{ }^{\circ} \mathrm{C}$ with $50 \pm 5 \%$ humidity and subjected to a 12-h light/dark cycle Food and water was available ad libitum prior to exercise. The NS extract was freshly dissolved in either distilled water and orally administered using a gavage with a dose of $2 \mathrm{~g} / \mathrm{kg} /$ day. In addition, NS extract was administered on the 21st day, $1 \mathrm{~h}$ prior to the initiation of swimming exercise. Distilled water was administered for control group.

Exhaustive swimming. A pool for exhaustive swimming was designed especially for rats. The system consisted of a glass chamber $(70 \mathrm{~cm}$ in height, $60 \mathrm{~cm}$ in length and $90 \mathrm{~cm}$ in width) filled with water to a height of $55 \mathrm{~cm}$. The pool was equipped with a heating system and an air pumping system. To prevent floating during swimming, water bubbles were produced by tubes connected to the air pumping system. The temperature of the water within the glass chamber was maintained at $36 \pm 1^{\circ} \mathrm{C}$ via a thermostatically controlled heater located at the base of the chamber. The rats were individually applied to forced-swimming until exhaustion, which was defined as failure to rise to the surface of the water to breathe for $7 \mathrm{sec}(28)$.

Analysis of blood and serum biochemical parameters. Blood was collected from the tail vein just before swimming and from the caudal vena cava following swimming. A Nova Stat Profile ${ }^{\circledR}$ pHOx Ultra Analyzer system (Nova Biomedical, Waltham, MA, USA) was used to measure $\mathrm{pH}$ and to quantify levels of partial pressure of carbon dioxide $\left(p \mathrm{CO}_{2}\right.$, the partial pressure of oxygen $\left(p \mathrm{O}_{2}\right)$ and oxygen saturation $\left(\mathrm{O}_{2 \mathrm{sat}}\right)$. In addition, the concentrations of glucose, lactate and hemoglobin $(\mathrm{Hb})$ were measured, along with hematocrit (Hct). Serum was immediately separated by centrifugation at $1,500 \mathrm{x} g$ for $10 \mathrm{~min}$ following swimming and stored at $-80^{\circ} \mathrm{C}$ until biochemical analysis. A Hitachi 7020 system (Hitachi Corporation, Tokyo, Japan) was used for analyses of total protein (TP), albumin, lactic dehydrogenase (LDH) and creatine kinase (CK) levels.
Analysis of glycogen contents in liver and gastrocnemius muscle (g. muscle) tissues. The liver and g. muscle were cut, weighed and homogenized in cold perchloric acid. The homogenate was centrifuged for $15 \mathrm{~min}$ at $15,000 \mathrm{x} g$ at $4^{\circ} \mathrm{C}$. The supernatant was carefully decanted. A standard glycogen (Sigma; St.Louis, MO, USA) and tissue extract were mixed with iodine-potassium iodide reagent for binding iodine to glycogen. The mixtures were measured by the SpectraMax M3 ELISA reader (Molecular Devices, LLC, Sunnyvale, CA, USA) at $460 \mathrm{~nm}$ wavelength.

Analysis of antioxidant defense. Levels of malondialdehyde (MDA) in serum, liver and g. muscle were measured with an OXI-TEK TBARS assay kit (Enzo Life Sciences Inc., Farmingdale, NY, USA). Reaction products were quantitated by measuring the absorbance at $532 \mathrm{~nm}$ according to the manufacturer's protocol. The levels of superoxide dismutase (SOD) in the serum, liver and g. muscle were quantitated using a SOD activity kit (cat. no. ADI-900-157, Enzo Life Sciences Inc.) by measuring the absorbance of the reaction products at $450 \mathrm{~nm}$. The total glutathione (tGSH) and oxidized glutathione (GSSG) levels were measured using a glutathione (total) detection kit (cat. no. ADI-900-160) from Enzo Life Sciences Inc. by measuring the absorbance of the reaction products at $405 \mathrm{~nm}$. The concentration of reduced glutathione (GSH) was calculated using the formula (GSH=tGSH-GSSG), which was provided in the kit protocol. The redox ratio was calculated using the formula GSH:GSSG=(tGSH-2GSSG)/GSSG, as described previously (29).

Statistical analysis. All data are reported as mean \pm standard error of the mean. Statistical significance was analyzed using the Student's $t$ test or, where applicable, two-way analysis of variance with Bonferroni post-hoc analysis for multiple group comparisons using GraphPad Prism software (version, 5.03; GraphPad Software Inc., La Jolla, CA, USA), and $\mathrm{P}<0.05$ was considered statistically significant.

\section{Results}

Effects of NS extract on the exhaustive swimming on rats. To evaluate the effect of NS seed extract on exercise durability, the exhaustive swimming test was performed using rats, which were pre-treated with either distilled water as a control or NS extracts for 21 days. As demonstrated in Fig. 1, the swimming time to exhaustion was significantly increased in the NS-treated group compared with control group $(\mathrm{P}<0.001$; $24.6 \%$ increase vs. control). These data indicated that NS seed extract may prolong the swimming time in rats.

Effects of NS seed extract on hemodynamic parameters following exhaustive swimming. Exhaustive swimming resulted in significant decreases of $\mathrm{pH}(\mathrm{P}<0.001), p \mathrm{O}_{2}(\mathrm{P}<0.001)$ and $\mathrm{O}_{2 \text { sat }}$ levels $(\mathrm{P}<0.001)$, but an increase of $p \mathrm{CO}_{2}$ level $(\mathrm{P}<0.001)$, when compared with the pre-swimming control group. However, these changes were significantly attenuated by pre-treatment with NS seed extract compared with the post-swimming control group $(\mathrm{P}<0.01)$. Hb and Hct levels were slightly increased $(\mathrm{P}>0.05)$ following swimming in the control group. Notably, in pre-swimming and post-swimming 
Table I. Effects of NS extract on blood biochemical parameters after forced-swimming.

\begin{tabular}{|c|c|c|c|c|}
\hline & \multicolumn{2}{|c|}{ Control } & \multicolumn{2}{|c|}{ NS } \\
\hline & Pre-swimming & Post-swimming & Pre-swimming & Post-swimming \\
\hline $\mathrm{pH}$ & $7.30 \pm 0.03$ & $7.09 \pm 0.03^{\mathrm{c}}$ & $7.30 \pm 0.02$ & $7.13 \pm 0.03^{\mathrm{e}}$ \\
\hline $\mathrm{Hb}(\mathrm{g} / \mathrm{dl})$ & $12.5 \pm 0.5$ & $13.0 \pm 0.4^{\mathrm{a}}$ & $15.5 \pm 0.4^{\mathrm{d}}$ & $15.9 \pm 0.4$ \\
\hline $\operatorname{Hct}(\%)$ & $42 \pm 1$ & $43 \pm 1^{\mathrm{a}}$ & $45 \pm 1^{\mathrm{d}}$ & $46 \pm 1$ \\
\hline$p \mathrm{CO}_{2}(\mathrm{mmHg})$ & $44.8 \pm 2.5$ & $78.7 \pm 4.9^{c}$ & $42.0 \pm 2.4$ & $68.2 \pm 3.0^{\mathrm{e}}$ \\
\hline$p \mathrm{O}_{2}(\mathrm{mmHg})$ & $58.6 \pm 3.0$ & $24.7 \pm 1.7^{\mathrm{c}}$ & $67.9 \pm 2.9^{\mathrm{a}}$ & $37.9 \pm 2.78^{\mathrm{e}}$ \\
\hline $\mathrm{O}_{2 \mathrm{Sat}}(\%)$ & $81.6 \pm 2.2$ & $33.0 \pm 4.9^{c}$ & $85.3 \pm 3.5$ & $46.4 \pm 3.9^{\mathrm{e}}$ \\
\hline $\mathrm{TP}(\mathrm{g} / \mathrm{dl})$ & $5.0 \pm 0.2$ & $6.4 \pm 0.3^{\mathrm{b}}$ & $4.7 \pm 0.2$ & $5.6 \pm 0.3^{d}$ \\
\hline Albumin (g/dl) & $2.9 \pm 0.2$ & $3.1 \pm 0.2$ & $2.6 \pm 0.1$ & $3.0 \pm 0.1$ \\
\hline
\end{tabular}

The data are reported as the mean \pm standard error of the mean $(n=10)$. Significance was measured via two-way analysis of variance. ${ }^{a} \mathrm{P}<0.05$, ${ }^{\mathrm{b}} \mathrm{P}<0.01$ and ${ }^{\mathrm{c}} \mathrm{P}<0.001$ vs. pre-swimming control group; ${ }^{\mathrm{d}} \mathrm{P}<0.05$, ${ }^{\mathrm{e}} \mathrm{P}<0.01$ and ${ }^{\mathrm{f}} \mathrm{P}<0.001$ vs. pre-swimming NS-treated group. Control, control group; NS, NS-treated group; $\mathrm{Hb}$, hemoglobin; Hct, hematocrit; $p \mathrm{CO}_{2}$, partial pressure of carbon dioxide; $p \mathrm{O}_{2}$, partial pressure of oxygen; $\mathrm{O}_{2 \text { sat }}$, oxygen saturation; TP, total protein.

of NS-pre-treated groups, these values had a tendency to increase compared with pre-swimming-control group, even though values in post-swimming group with pre-treatment of NS extract were not significantly changed compared with post-swimming control group ( $\mathrm{P}>0.05$; Table I). Finally, TP was markedly elevated in both control and NS-pre-treated groups following exhaustive swimming, when compared with pre-swimming control group. Moreover, there was no obvious difference in albumin levels between control and NS-treated groups following exhaustive swimming (Table I). These data indicated that NS seed extract could maintain the blood homeostasis following exhaustive swimming.

Effects of NS seed extract on fatigue-related serum biomarkers after exhaustive swimming. Fatigue can be evaluated by several important biochemical indicators, including glucose, lactate, LDH and CK. Exhaustive swimming caused depleted glucose, as an energy source, and the accumulation of lactate, LDH and CK (30). Thus, the authors examined the levels of fatigue-related blood biomarkers to test the anti-fatigue effect of NS seed extract. The exhaustive swimming led to a significant decrease in serum glucose level (from 127 to $103.2 \mathrm{mg} / \mathrm{dl}$ following exercise; $\mathrm{P}<0.001)$ but significant increases in lactate (3.5 to $5.5 \mathrm{mmol} / \mathrm{l} ; \mathrm{P}<0.001)$, LDH (202.4 to $811.6 \mathrm{IU} / \mathrm{l} ; \mathrm{P}<0.001)$ and $\mathrm{CK}(405.6$ to $1288.4 \mathrm{IU} / 1 ; \mathrm{P}<0.001)$ levels (Fig. 2) compared with the pre-swimming-control group. However, pre-treatment with NS extract significantly protected these exercise-induced alterations after exhaustive swimming (Fig. 2). The serum glucose level was significantly increased in the NS-treated group compared with control group following exhaustive swimming $(\mathrm{P}<0.01)$. Conversely, increased levels of serum lactate, $\mathrm{LDH}$ and $\mathrm{CK}$, due to exhaustive swimming, were significantly attenuated by pre-treatment of NS extract (lactate, $\mathrm{P}<0.05$; LDH and CK, $\mathrm{P}<0.001$; Fig. 2). Taken together, the authors also demonstrated the anti-fatigue effects of NS extract on exhaustive swimming from the analysis of typical fatigue-related indicators (e.g., glucose, lactate, LDH and $\mathrm{CK})$.

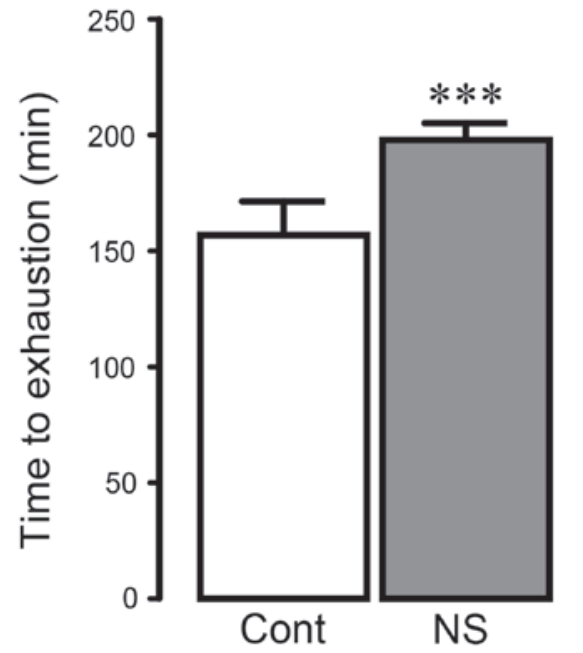

Figure 1. Effect of NS extract on the exhaustive swimming on rats. Time to exhaustion was measured in rats pre-treated with the distilled water as a control or NS extract. Data are expressed as the means \pm standard error of the mean ( $n=10$ per group). Significance was measured via paired Student's t-test. ${ }^{* * * *} \mathrm{P}<0.001$. NS, Nigella sativa; Cont, control group; NS, NS-treated group.

Effect of NS extract on glycogen contents in liver and muscle. Glycogen content, which is main storage form of glucose, is an integral determining factor in fatigue following exhaustive swimming. Many studies have reported that glycogen is significantly depleted in both liver and muscles during exhaustive swimming (31). In the present study, following exhaustive swimming, glycogen levels in liver and g. muscle were significantly diminished compared with pre-swimming-control group (21.5 and $11.5 \%$ decreases in liver and g. muscle vs. control; $\mathrm{P}<0.001$, respectively; Fig. 3). However, in NS extract-treated group, glycogen content was restored in both liver and $\mathrm{g}$. muscle (39.4 and 10.2\% increases in liver and g. muscle vs. swimming-control; $\mathrm{P}<0.001$, respectively). In particular, the liver glycogen in NS-pre-treated-swimming group was significantly increased compared with control group (9.5\% increase vs. control; $\mathrm{P}<0.05$; Fig. $3 \mathrm{~A})$. These results demonstrated that 
A

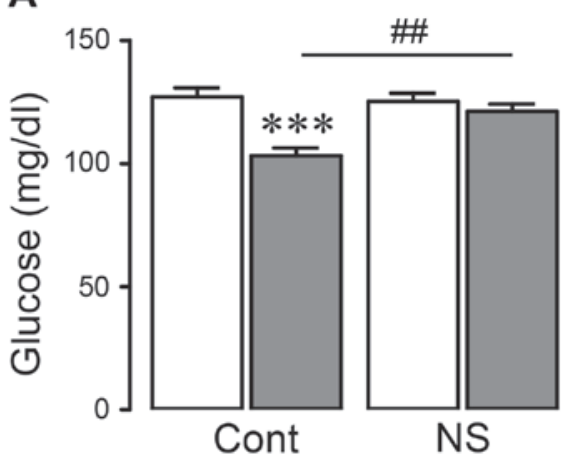

C

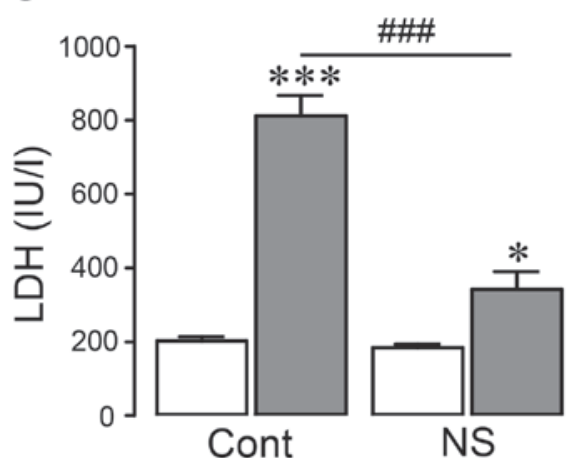

B

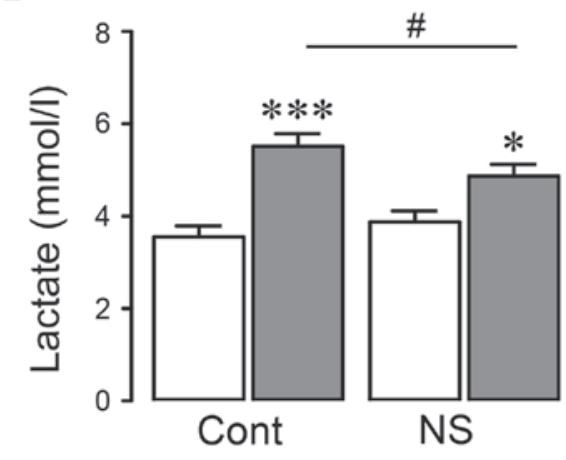

D

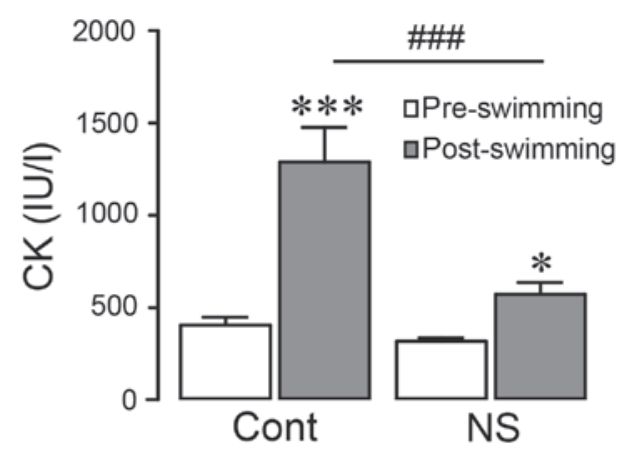

Figure 2. Effects of NS extract on serum biomarkers related to fatigue. Serum levels of (A) glucose, (B) lactate, (C) LDH and (D) CK were measured following the collection of blood just prior to and following swimming. The Cont group were treated with distilled water, while the experimental group was treated with NS extract. Data are expressed as the means \pm standard error of the mean ( $\mathrm{n}=10$ per group). ${ }^{*} \mathrm{P}<0.05,{ }^{* *} \mathrm{P}<0.01$ and ${ }^{* * * *} \mathrm{P}<0.001$, measured via Bonferroni post hoc test following two-way analysis of variance vs. the pre-swimming Cont; ${ }^{\#} \mathrm{P}<0.05,{ }^{\# \#} \mathrm{P}<0.01$ and ${ }^{\# \# \#} \mathrm{P}<0.001$, measured via Student's $\mathrm{t}$-test between post-swimming groups. NS, Nigella sativa; LDH, lactate dehydrogenase; CK, creatine kinase; Cont, control group; NS, NS-treated group.

A

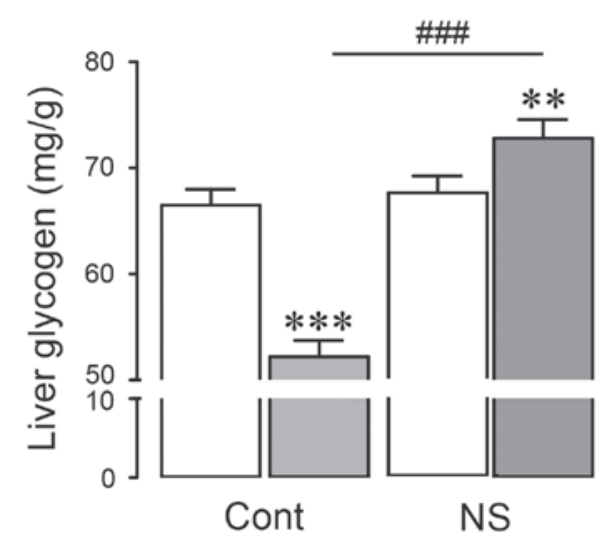

$\mathbf{B}$

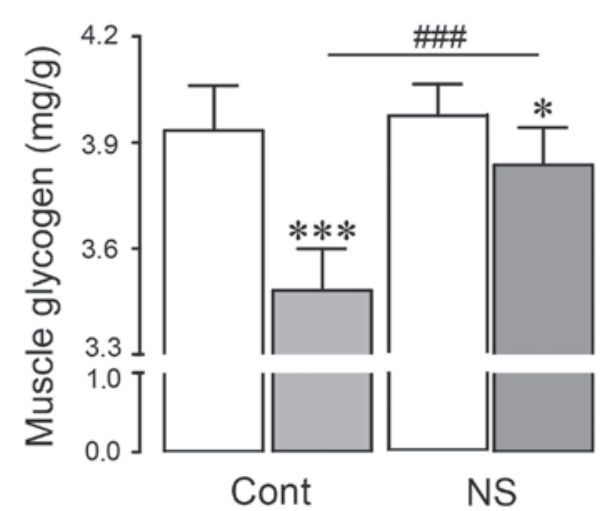

Figure 3. Effect of NS extract on glycogen contents in liver and muscle. Glycogen levels in (A) liver and (B) g. muscle were measured by enzyme-linked immunosorbent assay following the collection of liver and g. muscle tissues just prior to swimming or after swimming. Tissues were treated with distilled water (control) or NS extract. Data are expressed as the means \pm standard error of the mean ( $=10$ per group). ${ }^{*} \mathrm{P}<0.05,{ }^{* * *} \mathrm{P}<0.01$ and ${ }^{* * * *} \mathrm{P}<0.001$, measured via Bonferroni post hoc test following two-way analysis of variance vs. the pre-swimming Cont; ${ }^{\#} \mathrm{P}<0.05,{ }^{\# \# /} \mathrm{P}<0.01$ and ${ }^{\# \# \#} \mathrm{P}<0.001$, measured via Student's $\mathrm{t}$-test between post-swimming groups. NS, Nigella sativa; g. muscle, gastrocnemius muscle; Cont., control group; NS, NS-treated group.

NS extract administration may reserve glycogen in liver and g. muscle.

Effects of NS seed extract on antioxidant parameters in serum, liver and $g$. muscle. Oxidative stress occurs following exhaustive swimming, and subsequently may lead to pathology and clinical symptoms of fatigue (32). Therefore, the authors investigated the antioxidant activity of NS extract following exhaustive swimming by examining the SOD, MDA and serum redox ratio (GSH/GSSG) values as typical oxidative stress related parameters. Serum SOD level was significantly decreased in the swimming control-group, when compared with the control group $(4.9 \%$ decrease vs. control; $\mathrm{P}<0.05)$. As expected, in the NS extract-pre-treated group, serum SOD 
A

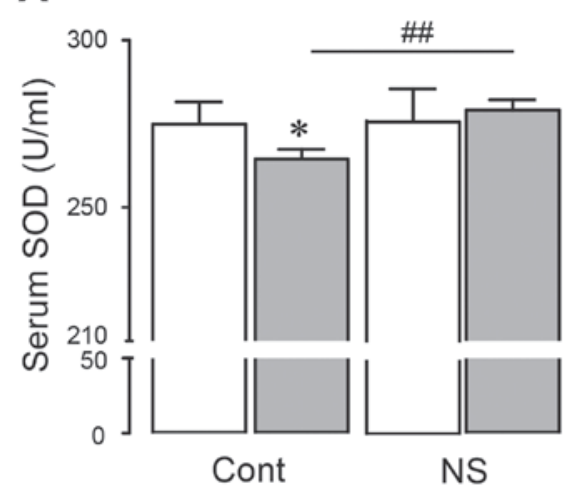

C

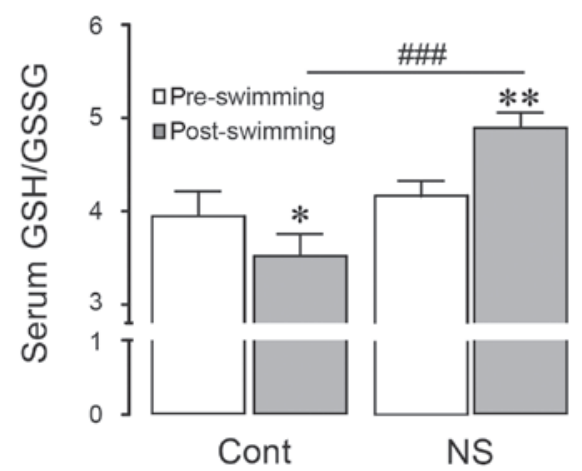

B

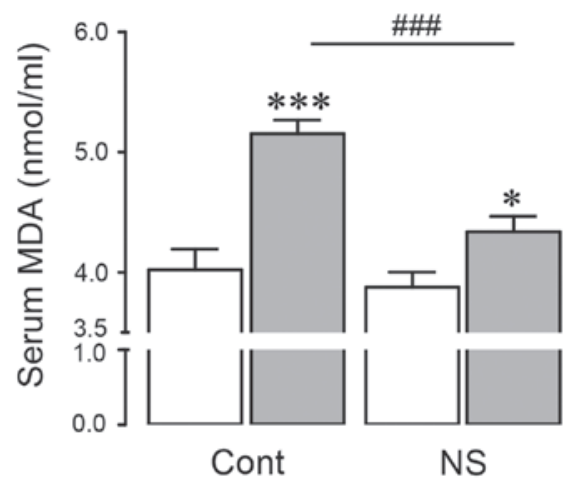

Figure 4. Effects of NS extract on serum antioxidant parameters. Serum levels of (A) SOD and (B) MDA were measured by enzyme-linked immunosorbent assay after collecting blood just prior to swimming or following swimming. Tissues were treated with distilled water (control) or NS extract. (C) The redox ratio (GSH/GSSG) was calculated following measuring serum GSH and GSSG. Data are expressed as the means \pm standard error of the mean ( $=10$ per group). ${ }^{*} \mathrm{P}<0.05,{ }^{* *} \mathrm{P}<0.01$ and ${ }^{* * *} \mathrm{P}<0.001$, measured via Bonferroni post hoc test following two-way analysis of variance vs. the pre-swimming Cont.; ${ }^{\#} \mathrm{P}<0.05$, ${ }^{\# \#} \mathrm{P}<0.01$ and ${ }^{\# \#} \mathrm{P}<0.001$, measured via Student's t-test between post-swimming groups. NS, Nigella sativa; SOD, superoxide dismutase; MDA, malondialdehyde; GSH, reduced glutathione; GSSG, oxidized glutathione; Cont, control group; NS, NS-treated group.

level was significantly increased compared with the swimming control-group following exhaustive swimming $(5.8 \%$ increase vs. swimming-control; $\mathrm{P}<0.01$; Fig. 4A). Serum MDA level was dramatically increased following exhaustive swimming $(28.1 \%$ increase vs. control; $\mathrm{P}<0.001)$. However, pre-treatment with NS extract inhibited the serum MDA level following exhaustive swimming (15.8\% decrease vs. swimming-control; $\mathrm{P}<0.001$; Fig. 4B). Similarly, the serum redox ratio (GSH:GSSG) was significantly decreased in the swimming-control group, when compared with the control group ( $10.8 \%$ decrease vs. control; $\mathrm{P}<0.05)$, but was increased in the exercise-NS extract-treated group (39.1\% increase vs. swimming-control; $\mathrm{P}<0.001$; Fig. 4C).

In addition, SOD levels in liver and g. muscle were significantly decreased compared with the control group following exhaustive swimming (21.9 and 6.0\% decreases in liver and g. muscle vs. control; $\mathrm{P}<0.001$, respectively; Fig. 5A and C). Notably, SOD levels in both tissues were significantly increased by pre-treatment of NS extract compared with the swimming-control-group (50.5 and 8.0\% increases in liver and g. muscle vs. swimming-control; $\mathrm{P}<0.001$, respectively; Fig. 5A and C). MDA levels in liver and g. muscle were significantly increased by pre-treatment of NS extract compared with the control group following exhaustive swimming (58.7 and 36.9\% increases, in liver and g. muscle vs. control; $\mathrm{P}<0.001$, respectively). However, when the NS extract was pre-treated, MDA levels in both tissues were significantly inhibited compared with exercise-control group (30.4 and $14.2 \%$ decreases in liver and g. muscle vs. swimming-control; $\mathrm{P}<0.001$, respectively; Fig. 5B and D). These data suggested that NS extract may have a beneficial role against oxidative stress to alleviate physical fatigue following exercise.

\section{Discussion}

NS seeds possess many pharmacological activities, and are especially well known for their potent antioxidant effects. Many previous studies have reported that NS seeds may reduce toxicity in a number of diseases including diabetes, neural disease, renal disease, cardiovascular disease and cancer, due to their antioxidant activities from both in vitro and in vivo approaches (6,33-36). Therefore, in the present study, the authors focused on determining the protective effects of NS seeds against exhaustive swimming-induced fatigue.

To evaluate the anti-fatigue effects of NS extract in rats, the authors performed the exhaustive swimming, which has been commonly used as a fatigue model (37). The results demonstrated that pre-treatment of NS extract significantly increased the swimming time to exhaustion (Fig. 1). Further, in hemodynamic parameters, NS extract may attenuate decreased values of $p \mathrm{O}_{2}$ and $\mathrm{O}_{2 \text { sat }}$ and decrease values of $p \mathrm{CO}_{2}$, resulting in impaired oxygen supply during fatigue (Table I). Thus, the 
A

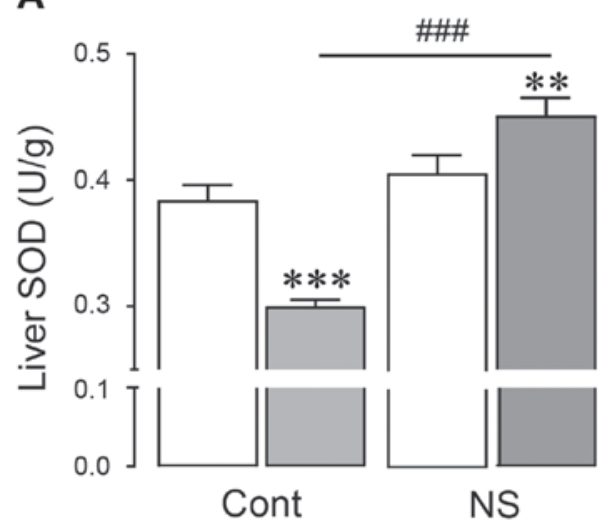

C

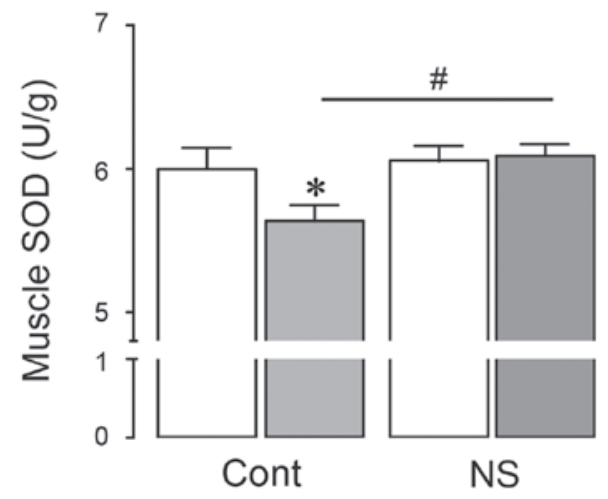

B

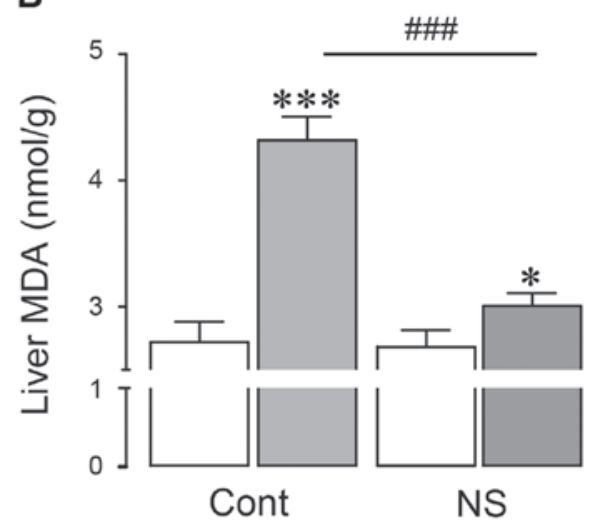

D

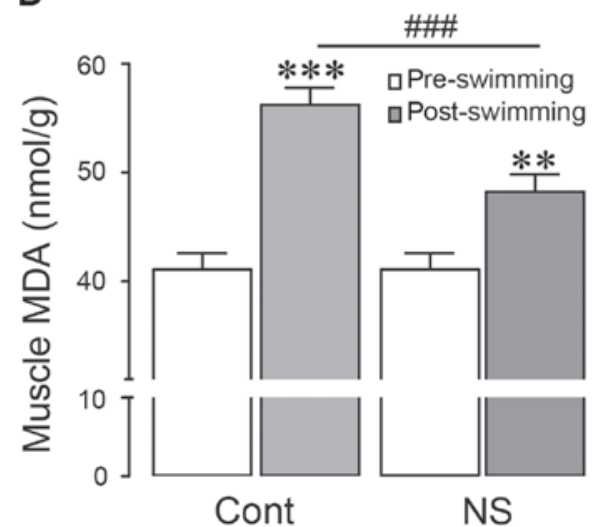

Figure 5. Effects of NS extract on antioxidant parameters in the liver and g. muscle tissues. (A and C) SOD and (B and D) MDA levels were measured by enzyme-linked immunosorbent assay following the collection of liver and $\mathrm{g}$. muscle tissues just before swimming or after swimming. Tissues were treated with distilled water (control) or NS extract. Data are expressed as the means \pm standard error of the mean ( $\mathrm{n}=10$ per group). ${ }^{*} \mathrm{P}<0.05$, ${ }^{* *} \mathrm{P}<0.01$ and ${ }^{* * *} \mathrm{P}<0.001$, measured via Bonferroni post hoc test following two-way analysis of variance vs. the pre-swimming cont.; ${ }^{\#} \mathrm{P}<0.05$, ${ }^{\# \prime} \mathrm{P}<0.01$ and ${ }^{\# \#} \mathrm{P}<0.001$, measured via Student's t-test between post-swimming groups. NS, Nigella sativa; SOD, superoxide dismutase; MDA, malondialdehyde; Cont, control group; NS, NS-treated group.

presented results indicated that NS extract may improve the swimming capacity against the fatigue.

Main causes of fatigue after exhaustive swimming are the depletion of energy sources and dysregulation of anti-oxidant defenses system (30). Regarding the depletion of energy sources, many studies have been demonstrated that energy sources, such as glucose and glycogen are depleted during exhaustive swimming, which in turn cause to physical fatigue (38). Here, it was observed that glycogen contents in both the liver and g. muscle were restored upon administration of NS extract (Figs. 2A and 5). In addition, under normal conditions, ATP, as an energy source, is produced by glycolysis (conversion of glycogen into glucose), which is, in turn, broken down into pyruvate (39). However, the muscles obtain energy from anaerobic glycolysis (conversion of pyruvate to lactate) during intense exercise. This intense exercise leads to accumulation of lactate, and lactate then drops the blood and muscle $\mathrm{pH}$, and consequently, fatigue is occurred (40). The results demonstrated that levels of serum glucose, lactate and LDH (key enzyme for lactate production) were increased following exhaustive swimming. Notably, these increases were dramatically attenuated when NS extract pre-treated (Fig. 2B-D). In addition, it was demonstrated that glycogen contents in liver and $g$ muscle tissues were dramatically preserved when pre-treated NS extract following exhaustive swimming (Fig. 3). Therefore, the authors suggested that NS extracts may contribute to fatigue retardation through preservation of glycogen content and reduction of lactate accumulation.

Secondly, the other important factor in fatigue is dysregulation of the antioxidant defense system (30). Exhaustive swimming may release reactive oxygen species (ROS) due to increased oxygen consumption, resulting in the development of fatigue. Therefore, MDA (an oxidative degradation product of cell membrane lipids) and SOD (major component of anti-oxidant defense system) are considered to be physiological markers relevant for fatigue. In the present study, the results revealed that elevated MDA levels and decreased levels of SOD, as well as the serum redox ratio (GSH:GSSG) in liver and $\mathrm{g}$. muscle tissues were completely reversed by pre-treatment of NS extract (Fig. 4). These findings suggested that anti-fatigue effect of NS extract is involved in the modulation of oxidative stress following exhaustive swimming.

In conclusion, the current study demonstrated that NS may be able to alleviate the physical fatigue by inhibiting energy depletion and oxidative stress. The authors further propose that NS may be a potential strategy for prevention and treatment of physical fatigue. 


\section{Acknowledgements}

The current research was supported by the research funds of Korean Ministry of Science (grant no. 2011-0013872) and the Brain Korea Plus program. The authors would like to thank Proofreading Service Center of Chonbuk National University and e-World Editing Ltd. for proofreading of the manuscript.

\section{References}

1. Hajhashemi V, Ghannadi A and Jafarabadi H: Black cumin seed essential oil, as a potent analgesic and antiinflammatory drug. Phytother Res 18: 195-199, 2004.

2. Amin B and Hosseinzadeh H: Black cumin (Nigella sativa) and its active constituent, thymoquinone: An overview on the analgesic and anti-inflammatory effects. Planta Med 82: 8-16, 2016.

3. Abdel-Fattah AM, Matsumoto $\mathrm{K}$ and Watanabe $\mathrm{H}$ : Antinociceptive effects of Nigella sativa oil and its major component, thymoquinone, in mice. Eur J Pharmacol 400: 89-97, 2000.

4. Houghton PJ, Zarka R, de las Heras B and Hoult JR: Fixed oil of Nigella sativa and derived thymoquinone inhibit eicosanoid generation in leukocytes and membrane lipid peroxidation. Planta Med 61: 33-36, 1995.

5. Burits $\mathrm{M}$ and Bucar F: Antioxidant activity of Nigella sativa essential oil. Phytother Res 14: 323-328, 2000.

6. Singh S, Das SS, Singh G, Schuff C, de Lampasona MP and Catalán CA: Composition, in vitro antioxidant and antimicrobial activities of essential oil and oleoresins obtained from black cumin seeds (Nigella sativa L.). Biomed Res Int 2014: 918209, 2014.

7. Kacem R and Meraihi Z: Effects of essential oil extracted from Nigella sativa (L.) seeds and its main components on human neutrophil elastase activity. Yakugaku Zasshi 126: 301-305, 2006.

8. Woo CC, Kumar AP, Sethi G and Tan KH: Thymoquinone: Potential cure for inflammatory disorders and cancer. Biochem Pharmacol 83: 443-451, 2012.

9. Ghannadi A, Hajhashemi V and Jafarabadi H: An investigation of the analgesic and anti-inflammatory effects of Nigella sativa seed polyphenols. J Med Food 8: 488-493, 2005.

10. Cikman O, Ozkan A, Aras AB, Soylemez O, Alkis H, Taysi S and Karaayvaz M: Radioprotective effects of Nigella sativa oil against oxidative stress in liver tissue of rats exposed to total head irradiation. J Invest Surg 27: 262-266, 2014.

11. Assayed ME: Radioprotective effects of black seed (Nigella sativa) oil against hemopoietic damage and immunosuppression in gamma-irradiated rats. Immunopharmacol Immunotoxicol 32: 284-296, 2010.

12. Ebru U, Burak U, Yusuf S, Reyhan B, Arif K, Faruk TH, Emin M, Aydin K, Atilla II, Semsettin S and Kemal E: Cardioprotective effects of Nigella sativa oil on cyclosporine A-induced cardiotoxicity in rats. Basic Clin Pharmacol Toxicol 103: 574-580, 2008.

13. Nagi MN and Mansour MA: Protective effect of thymoquinone against doxorubicin-induced cardiotoxicity in rats: A possible mechanism of protection. Pharmacol Res 41: 283-289, 2000.

14. Mahmoud MR, El-Abhar HS and Saleh S: The effect of Nigella sativa oil against the liver damage induced by Schistosoma mansoni infection in mice. J Ethnopharmacol 79: 1-11, 2002.

15. Moriura T, Matsuda $\mathrm{H}$ and Kubo M: Pharmacological study on Agkistrodon blomhoffii blomhoffii BOIE. V. anti-fatigue effect of the $50 \%$ ethanol extract in acute weight-loaded forced swimming-treated rats. Biol Pharm Bull 19: 62-66, 1996.

16. Kim KM, Yu KW, Kang DH, Koh JH, Hong BS and Suh HJ: Anti-stress and anti-fatigue effects of fermented rice bran. Biosci Biotechnol Biochem 65: 2294-2296, 2001.

17. Cochrane GD, Rizvi S, Abrantes AM, Crabtree B, Cahill J and Friedman JH: The association between fatigue and apathy in patients with either Parkinson's disease or multiple sclerosis. Parkinsonism Relat Disord 21: 1093-1095, 2015.

18. Theoharides TC: Atopic conditions in search of pathogenesis and therapy. Clin Ther 35: 544-547, 2013.
19. Kluger BM, Rakowski D, Christian M, Cedar D, Wong B, Crawford J, Uveges K, Berk J, Abaca E, Corbin L and Garvan C: Randomized, controlled trial of acupuncture for fatigue in parkinson's disease. Mov Disord 31: 1027-1032, 2016.

20. Hao G, Zhang C, Cao W and Hao J: Effects of intragastric administration of five oyster components on endurance exercise performance in mice. Pharm Biol 52: 723-728, 2014.

21. Zhang XL, Ren F, Huang W, Ding RT, Zhou QS and Liu XW: Anti-fatigue activity of extracts of stem bark from Acanthopanax senticosus. Molecules 16: 28-37, 2011.

22. Alessio HM: Exercise-induced oxidative stress. Med Sci Sports Exerc 25: 218-224, 1993.

23. Lee SP, Mar GY and Ng LT: Effects of tocotrienol-rich fraction on exercise endurance capacity and oxidative stress in forced swimming rats. Eur J Appl Physiol 107: 587-595, 2009.

24. Castro-Marrero J, Sáez-Francàs N, Santillo D and Alegre J: Treatment and management of chronic fatigue syndrome/myalgic encephalomyelitis: All roads lead to Rome. Br J Pharmacol 174: 345-369, 2017.

25. Korsen M, Kunz R, Schminke U, Runge U, Kohlmann T and Dressel A: Dalfampridine effects on cognition, fatigue, and dexterity. Brain Behav 7: e00559, 2016.

26. Tharakan B, Dhanasekaran M and Manyam BV: Antioxidant and DNA protecting properties of anti-fatigue herb Trichopus zeylanicus. Phytother Res 19: 669-673, 2005.

27. Le PM, Benhaddou-Andaloussi A, Elimadi A, Settaf A, Cherrah Y and Haddad PS: The petroleum ether extract of Nigella sativa exerts lipid-lowering and insulin-sensitizing actions in the rat. J Ethnopharmacol 94: 251-259, 2004.

28. Oh TW, Oh TW and Ohta F: Dose-dependent effect of capsaicin on endurance capacity in rats. Br J Nutr 90: 515-520, 2003.

29. Behr J, Maier K, Degenkolb B, Krombach F and Vogelmeier C: Antioxidative and clinical effects of high-dose $\mathrm{N}$-acetylcysteine in fibrosing alveolitis. Adjunctive therapy to maintenance immunosuppression. Am J Respir Crit Care Med 156: 1897-1901, 1997.

30. Norheim KB, Jonsson G and Omdal R: Biological mechanisms of chronic fatigue. Rheumatology (Oxford) 50: 1009-1018, 2011.

31. Shang H, Cao S, Wang J, Zheng H and Putheti R: Glabridin from Chinese herb licorice inhibits fatigue in mice. Afr J Tradit Complement Altern Med 7: 17-23, 2009.

32. Wu C, Chen R, Wang XS, Shen B, Yue W and Wu Q: Antioxidant and anti-fatigue activities of phenolic extract from the seed coat of Euryale ferox Salisb. and identification of three phenolic compounds by LC-ESI-MS/MS. Molecules 18: 11003-11021, 2013.

33. Adam GO, Rahman MM, Lee SJ, Kim GB, Kang HS, Kim JS and Kim SJ: Hepatoprotective effects of Nigella sativa seed extract against acetaminophen-induced oxidative stress. Asian Pac J Trop Med 9: 221-227, 2016

34. Magdy MA, Hanan el-A and Nabila el-M: Thymoquinone: Novel gastroprotective mechanisms. Eur J Pharmacol 697: 126-131, 2012.

35. Hosseinzadeh H, Parvardeh S, Asl MN, Sadeghnia HR and Ziaee T: Effect of thymoquinone and Nigella sativa seeds oil on lipid peroxidation level during global cerebral ischemia-reperfusion injury in rat hippocampus. Phytomedicine 14: 621-627, 2007.

36. Al Wafai RJ: Nigella sativa and thymoquinone suppress cyclooxygenase- 2 and oxidative stress in pancreatic tissue of streptozotocin-induced diabetic rats. Pancreas 42: 841-849, 2013.

37. Tang W, Zhang Y, Gao J, Ding X and Gao S: The anti-fatigue effect of 20(R)-ginsenoside Rg3 in mice by intranasally administration. Biol Pharm Bull 31: 2024-2027, 2008.

38. Wang L, Zhang HL, Lu R, Zhou YJ, Ma R, Lv JQ, Li XL, Chen LJ and Yao Z: The decapeptide CMSO01 enhances swimming endurance in mice. Peptides 29: 1176-1182, 2008.

39. Westerblad H, Bruton JD and Katz A: Skeletal muscle: Energy metabolism, fiber types, fatigue and adaptability. Exp Cell Res 316: 3093-3099, 2010.

40. Cairns SP: Lactic acid and exercise performance: Culprit or friend? Sports Med 36: 279-291, 2006. 